\title{
Strictly singular inclusions of rearrangement invariant spaces and Rademacher spaces
}

\author{
by \\ Sergei V. Astashkin (Samara), Francisco L. Hernández (Madrid) \\ and Evgeni M. Semenov (Voronezh)
}

\begin{abstract}
If $G$ is the closure of $L_{\infty}$ in $\exp L_{2}$, it is proved that the inclusion between rearrangement invariant spaces $E \subset F$ is strictly singular if and only if it is disjointly strictly singular and $E \not \supset G$. For any Marcinkiewicz space $M(\varphi) \subset G$ such that $M(\varphi)$ is not an interpolation space between $L_{\infty}$ and $G$ it is proved that there exists another Marcinkiewicz space $M(\psi) \subsetneq M(\varphi)$ with the property that the $M(\psi)$ and $M(\varphi)$ norms are equivalent on the Rademacher subspace. Applications are given and a question of Milman answered.
\end{abstract}

1. Introduction. A linear operator between two Banach spaces $E$ and $F$ is called strictly singular (SS for short), or Kato, if it fails to be an isomorphism on any infinite-dimensional subspace (cf. [LT1, 2.c.2]). The class of all strictly singular operators is a well-known closed operator ideal with important applications. A weaker notion for Banach lattices, introduced in $[\mathrm{HR}]$, is the following: a bounded operator $A$ from a Banach lattice $E$ to a Banach space $F$ is said to be disjointly strictly singular (DSS for short) if there is no disjoint sequence of non-null vectors $\left\{x_{n}\right\}_{n=1}^{\infty}$ in $E$ such that the restriction of $A$ to the subspace $\left[x_{n}\right]$ spanned by the vectors $\left\{x_{n}\right\}$ is an isomorphism. This is a useful tool in comparing structures of rearrangement invariant spaces (cf. [HR], [GHSS]).

This paper deals with the strict singularity of inclusions $E \subset F$ between rearrangement invariant (r.i.) function spaces $E$ and $F$ on the interval $[0,1]$. That means that the norms of $E$ and $F$ are non-equivalent on any (closed) infinite-dimensional subspace of $E$.

The canonical inclusion $L_{\infty} \subset E$ is always strictly singular for any r.i. space $E \neq L_{\infty}([\mathrm{N}])$, and the case of $L_{p}$-spaces is Grothendieck's classical result. Furthermore, this property characterizes the space $L_{\infty}$ among all r.i.

2000 Mathematics Subject Classification: Primary 46E30.

Key words and phrases: rearrangement invariant spaces, strict singularity, Rademacher subspace. 
spaces ([GHSS]). Concerning the right extreme inclusion $E \subset L_{1}$, its strict singularity has been characterized in [HNS] by the condition that the r.i. space $E$ does not contain the space $G$, the closure of $L_{\infty}$ in the exponential Orlicz space $\exp L_{2}$. Recall that Rodin and Semenov [RS] (see also [LT2]) proved that the condition $E \supset G$ determines precisely the r.i. spaces $E$ for which the Rademacher function system $\left\{r_{k}\right\}$ is equivalent to the canonical basis of $\ell_{2}$.

One of the aims of this article is to give a complete characterization of the strict singularity of inclusions between arbitrary r.i. spaces in terms of disjoint strict singularity. More precisely, it is proved in Section 3 (Theorem 2) that the inclusion $E \subset F$ is strictly singular if and only if it is disjointly strictly singular and the norms of these spaces are not equivalent on the Rademacher subspace $\left[r_{n}\right]$. This extends some previous results given in [HNS].

In [RS] the following result was proved for the class of r.i. spaces contained in $G$ : Under some additional assumptions, the equivalence of the norms in two r.i. spaces $E$ and $F$ of this class on the Rademacher subspace, i.e.,

$$
\left\|\sum c_{k} r_{k}\right\|_{E} \asymp\left\|\sum c_{k} r_{k}\right\|_{F},
$$

implies the coincidence of $E$ and $F$ up to equivalence of norms, i.e., $E=F$. More recently in $[\mathrm{A}]$ this result was obtained under a weaker assumption: the r.i. spaces $E$ and $F$ have to be interpolation spaces between $L_{\infty}$ and $G$. It turns out that this interpolation assumption is actually a necessary condition for the above statement to hold. Theorem 9 in Section 4 shows that for any Marcinkiewicz space $M(\varphi) \subset G$ such that $M(\varphi)$ is not an interpolation space between $L_{\infty}$ and $G$, there exists another Marcinkiewicz space $M(\psi) \subsetneq M(\varphi)$ with the property that the $M(\psi)$-norm and the $M(\varphi)$-norm are equivalent on the Rademacher subspace $\left[r_{n}\right]$. Also a criterion for the strict singularity of inclusions between Lorentz spaces $\Lambda(\varphi)$ and Marcinkiewicz spaces $M(\psi)$ is given (Theorem 11). In particular, for the class of all proper Lorentz spaces $\Lambda(\varphi)$ which do not contain $G$, the norms in $\Lambda(\varphi)$ and in the associated Marcinkiewicz space $M(\varphi)$ on the Rademacher subspace are never equivalent.

The last part of the paper contains some applications. In particular, we answer in the negative a question of V. Milman [Mi], showing that the r.i. spaces $E=L \log ^{\lambda} L$ and $F=L_{1}$ satisfy the following conditions: the inclusion $E \subset F$ is not strictly singular and any infinite-dimensional subspace of $E$ on which the norms of $E$ and $F$ are equivalent is an uncomplemented subspace of $E$ (Theorem 16 ). We also prove that any disjointly strictly singular inclusion between r.i. spaces is weakly compact.

Some results of this paper have been announced in $[\mathrm{SH}]$. 
2. Notation and definitions. Recall that a Banach function space $E$ of measurable functions on $[0,1]$ is called rearrangement invariant (r.i. for short) or symmetric (cf. [LT2, 2.a.1], [KPS, 2.4.1]) if

- $|x(t)| \leq|y(t)|$ for all $t \in[0,1]$ and $y \in E$ imply $x \in E$ and $\|x\|_{E} \leq$ $\|y\|_{E}$

- if $x$ and $y$ are equimeasurable and $y \in E$, then $x \in E$ and $\|x\|_{E}=\|y\|_{E}$.

As usual we assume that every r.i. space $E$ is separable or isomorphic to the conjugate space of some separable space. If $E$ is an r.i. space then $L_{\infty} \subset E \subset L_{1}$ and $\|x\|_{L_{1}} \leq\|x\|_{E} \leq\|x\|_{L_{\infty}}$ for each $x \in L_{\infty}$, assuming $\left\|\chi_{(0,1)}\right\|_{E}=1$.

Recall some important classes of r.i. spaces. If $M$ is a positive convex function on $[0, \infty)$ with $M(0)=0$, then the Orlicz space $L_{M}$ consists of all measurable functions on $[0,1]$ for which

$$
\|x\|_{L_{M}}=\inf \left\{s>0: \int_{0}^{1} M(|x(t)| / s) d t \leq 1\right\} .
$$

A remarkable example is the Orlicz space $L_{N}$ generated by the function $N(u)=e^{u^{2}}-1$. The space $L_{N}$ is non-separable and we will denote by $G$ the closure of $L_{\infty}$ in $L_{N}$. Another two special Orlicz spaces that will be considered here are generated by the functions $M(u)=e^{u^{\lambda}}-1$ and $M(u)=u \log ^{\lambda}(1+u)$, for $\lambda>0$, and denoted by $\exp L_{\lambda}$ and $L \log ^{\lambda} L$.

Let $\Omega$ be the set of all increasing concave functions on $[0,1]$ with $\varphi(0)=0$ and $\varphi(1)=1$. Each $\varphi \in \Omega$ generates the Lorentz space $\Lambda(\varphi)$ endowed with the norm

$$
\|x\|_{\Lambda(\varphi)}=\int_{0}^{1} x^{*}(t) d \varphi(t),
$$

and the Marcinkiewicz space $M(\varphi)$ with

$$
\|x\|_{M(\varphi)}=\sup _{0<\tau \leq 1} \frac{\varphi(\tau)}{\tau} \int_{0}^{\tau} x^{*}(t) d t
$$

where $x^{*}(t)$ is the decreasing rearrangement of $|x(t)|$. For any $\varphi \in \Omega$ we have $\Lambda(\varphi) \subset M(\varphi)$ and $\|x\|_{M(\varphi)} \leq\|x\|_{\Lambda(\varphi)}$ for every $x \in \Lambda(\varphi)$. The spaces $\Lambda(\varphi)$ and $M(\varphi)$ coincide up to equivalence of norms if and only if $\varphi(+0)>0$ or $\lim _{t \rightarrow 0} \varphi(t) / t<\infty$.

Recall that the fundamental function $\varphi_{E}$ of a r.i. space $E$ is defined by $\varphi_{E}(t)=\left\|\chi_{[0, t]}\right\|_{E}$ for $0 \leq t \leq 1$. The function $\varphi_{E}$ is quasi-concave, i.e., $\varphi_{E}(t)$ and $t / \varphi_{E}(t)$ increase on $(0,1]$. Up to equivalence of norms, $\varphi_{E}$ is a concave function. In that case $\Lambda\left(\varphi_{E}\right) \subset E \subset M\left(\varphi_{E}\right)$ and $\|x\|_{M\left(\varphi_{E}\right)} \leq\|x\|_{E} \leq$ $\|x\|_{\Lambda(\varphi)}$ for every $x \in \Lambda\left(\varphi_{E}\right)$. The fundamental function $\varphi_{E}(t)$ is continuous for $t \in(0,1]$. The condition $\varphi_{E}(+0)>0$ is necessary and sufficient for the 
coincidence of the spaces $E$ and $L_{\infty}$ up to equivalence of norms. If two r.i. spaces $E$ and $F$ coincide as sets then (by the closed graph theorem) the norms $\|\cdot\|_{E}$ and $\|\cdot\|_{F}$ are equivalent, and we write $E=F$.

Let $r_{k}(t)=\operatorname{sign}\left(\sin 2^{k} \pi t\right), k \in \mathbb{N}$, be the Rademacher functions on $[0,1]$. It was proved in [RS] (see also [LT2, Thm. 2.b.4]) that for an r.i. space $E$ the Khinchin inequality

$$
\left\|\sum_{k=1}^{\infty} c_{k} r_{k}\right\|_{E} \leq M\left\|\left\{c_{k}\right\}\right\|_{\ell_{2}}
$$

is valid, for some constant $M>0$, if and only if $E \supset G$. It follows immediately that for r.i. spaces $E$ and $F$ with $E \subset F$ the inclusion $E \subset F$ is not SS provided that $E \supset G$.

The proofs of some statements of this article will make use of interpolation methods. Therefore we recall some concepts and results in the r.i. setting.

Let $(E, F)$ be a pair of r.i. spaces and $x \in E+F$. The Peetre's $K$ functional is defined as

$$
K(t, x, E, F)=\inf \left\{\|u\|_{E}+t\|v\|_{F}: x=u+v\right\}
$$

for every $t>0$. Every Banach lattice $\Phi$ on $[0, \infty)$ such that $\min (1, t) \in \Phi$ generates the space $(E, F)_{\Phi}^{K}$ of the real interpolation method endowed with the norm

$$
\|x\|_{(E, F)_{\Phi}^{K}}:=\|K(\cdot, x, E, F)\|_{\Phi} .
$$

The space $(E, F)_{\Phi}^{K}$ has the interpolation property with respect to the pair $(E, F)$, i.e., every linear operator $A$ bounded in $E$ and $F$ is also bounded in $(E, F)_{\Phi}^{K}$ and $\|A\|_{(E, F)_{\Phi}^{K}} \leq \max \left(\|A\|_{E},\|A\|_{F}\right)$. In the classical case of $\Phi$ being the lattice on $[0, \infty)$ with the norm

$$
\|z\|_{\Phi}=\left(\int_{0}^{\infty}\left(t^{-\theta}|z(t)|\right)^{p} \frac{d t}{t}\right)^{1 / p},
$$

where $\theta \in(0,1)$ and $p \in[1, \infty]$ (with the usual modification for $p=\infty$ ), the interpolation spaces $(E, F)_{\Phi}^{K}$ are denoted by $(E, F)_{\theta, p}$.

We will denote by $I(E, F)$ the set of all interpolation spaces with respect to the pair $(E, F)$. If, for any $x, y \in E+F$ with $K(t, x, E, F) \leq K(t, y, E, F)$ for every $t>0$, there exists a linear operator $A$ bounded in $E$ and $F$ and such that $x=A y$, then the set $I(E, F)$ is described by the real interpolation method, in the sense that for each space $Q \in I(E, F)$ there exists a Banach lattice $\Phi$ such that $\|x\|_{Q}=\|K(\cdot, x, E, F)\|_{\Phi}($ cf. $[\mathrm{BK}])$.

If $f(x)$ and $g(x)$ are positive functions on some set $A$, we shall write $f \asymp g$ if there exists $C>0$ such that $C^{-1} f(x) \leq g(x) \leq C f(x)$ for every $x \in A$. 
We refer to the monographs [LT2] and [KPS] for the above results on r.i. spaces and to $[\mathrm{BK}]$ and $[\mathrm{BL}]$ for those on interpolation spaces.

3. Strict singularity via disjoint strict singularity. Given an r.i. space $E$ on $[0,1]$, we denote by $E_{0}$ the closure of $L_{\infty}$ in $E$. The space $E_{0}$ is always separable, except for $E=L_{\infty}$.

Proposition 1. Let $E$ and $F$ be r.i. spaces with $E \subset F$. Then the inclusion $E \subset F$ is disjointly strictly singular if and only if the inclusion $E_{0} \subset F$ is disjointly strictly singular.

Proof. The "only if" part is evident. Assume that the inclusion $E \subset F$ is not DSS. Thus there exist a disjoint sequence $\left\{x_{k}\right\}_{k=1}^{\infty}$ in $E$ and $M>0$ such that

$$
\left\|\sum_{k=1}^{n} c_{k} x_{k}\right\|_{E} \leq M\left\|\sum_{k=1}^{n} c_{k} x_{k}\right\|_{F}
$$

for every $n \in \mathbb{N}$ and $c_{k} \in \mathbb{R}$. We consider separately the cases (i) $E \subset F_{0}$ and (ii) $E \not \subset F_{0}$.

In case (i) we have $x_{k} \in F_{0}$ for $k \in \mathbb{N}$. Clearly we can assume $\left\|x_{k}\right\|_{F}=1$ for $k \in \mathbb{N}$. It is well known that $\lim _{\text {meas } A \rightarrow 0}\left\|x \chi_{A}\right\|_{F}=0$ for any $x \in F_{0}$ (cf. [KPS, 2.4.5]). Hence there exists a sequence $\left\{A_{k}\right\}_{k=1}^{\infty}$ of subsets of $[0,1]$ with $A_{k} \subset \operatorname{supp} x_{k}$ such that $y_{k}=x_{k} \chi_{A_{k}} \in L_{\infty} \subset E_{0}$ for $k \in \mathbb{N}$ and

$$
\sum_{k=1}^{\infty}\left\|x_{k}-y_{k}\right\|_{F}<\frac{1}{2}
$$

Now, by a stability result [LT1, Prop. 1.a.9], the sequences $\left\{x_{k}\right\}$ and $\left\{y_{k}\right\}$ are equivalent in $F$ and

$$
\left\|\sum_{k=1}^{n} c_{k} x_{k}\right\|_{F} \leq 2\left\|\sum_{k=1}^{n} c_{k} y_{k}\right\|_{F}
$$

for every $n \in \mathbb{N}$ and $c_{k} \in \mathbb{R}$. Since $\left|\sum_{k=1}^{n} c_{k} y_{k}\right| \leq\left|\sum_{k=1}^{n} c_{k} x_{k}\right|$, we have

$$
\left\|\sum_{k=1}^{n} c_{k} y_{k}\right\|_{E} \leq\left\|\sum_{k=1}^{n} c_{k} x_{k}\right\|_{E} \leq M\left\|\sum_{k=1}^{n} c_{k} x_{k}\right\|_{F} \leq 2 M\left\|\sum_{k=1}^{n} c_{k} y_{k}\right\|_{F}
$$

for any $n \in \mathbb{N}$ and $c_{k} \in \mathbb{R}$. Therefore the norms $\|\cdot\|_{E}$ and $\|\cdot\|_{F}$ are equivalent on the span of $\left\{y_{k}\right\}$ in $E_{0}$ and the inclusion $E_{0} \subset F$ is not DSS.

(ii) Consider now the case $E \not \subset F_{0}$. Since $E_{0} \subset F_{0}$ we have $E \backslash E_{0} \not \subset F_{0}$ and $\left(E \backslash E_{0}\right) \cap\left(F \backslash F_{0}\right) \neq \emptyset$. Choose $z=z^{*} \in\left(E \backslash E_{0}\right) \cap\left(F \backslash F_{0}\right)$. Then

$$
d_{E}\left(z, L_{\infty}\right)=a>0, \quad d_{F}\left(z, L_{\infty}\right)=b>0
$$

where $d_{E}\left(z, L_{\infty}\right)=\inf \left\{\|z-u\|_{E}: u \in L_{\infty}\right\}$. Since we have $\left\|z \chi_{(0, \tau)}\right\|_{E}=$ 
$\lim _{\varepsilon \rightarrow 0}\left\|z \chi_{(\varepsilon, \tau)}\right\|_{E}$ and $\left\|z \chi_{(0, \tau)}\right\|_{E} \geq a$ for $0<\tau \leq 1$, it follows that

$$
\lim _{\varepsilon \rightarrow 0}\left\|z \chi_{(\varepsilon, \tau)}\right\|_{E} \geq a \text {. }
$$

Similarly we have $\lim _{\varepsilon \rightarrow 0}\left\|z \chi_{(\varepsilon, \tau)}\right\|_{F} \geq b$ for $0<\tau \leq 1$. Hence we can construct a sequence $\tau_{k} \downarrow 0$ such that

$$
\left\|z \chi_{\left(\tau_{k+1}, \tau_{k}\right)}\right\|_{E} \geq a / 2 \text { and }\left\|z \chi_{\left(\tau_{k+1}, \tau_{k}\right)}\right\|_{F} \geq b / 2
$$

for every natural $k$. Let $z_{k}:=z \chi_{\left(\tau_{k+1}, \tau_{k}\right)}$ for $k \in \mathbb{N}$. Clearly, $z_{k} \in L_{\infty} \subset$ $E_{0} \subset F_{0}$ and

$$
\frac{a}{2} \max _{k \in \mathbb{N}}\left|c_{k}\right| \leq\left\|\sum_{k=1}^{\infty} c_{k} z_{k}\right\|_{E} \leq\|z\|_{E} \max _{k \in \mathbb{N}}\left|c_{k}\right|
$$

and

$$
\frac{b}{2} \max _{k \in \mathbb{N}}\left|c_{k}\right| \leq\left\|\sum_{k=1}^{\infty} c_{k} z_{k}\right\|_{F} \leq\|z\|_{F} \max _{k \in \mathbb{N}}\left|c_{k}\right|,
$$

for any sequence $\left\{c_{k}\right\} \in c_{0}$. Hence the sequence $\left\{z_{k}\right\}_{k=1}^{\infty}$ is equivalent in $E_{0}$ and in $F_{0}$ to the canonical basis of $c_{0}$. Consequently, the inclusion $E_{0} \subset F$ is not DSS.

Recall that $G$ is the closure of $L_{\infty}$ in $\exp L_{2}$. We can now prove the main result of this section.

TheOREM 2. Let $E$ and $F$ be r.i. spaces with $E \subset F$. The inclusion $E \subset F$ is strictly singular if and only if it is disjointly strictly singular and $E \not \supset G$.

Proof. The case of $E$ separable has been proved in [HNS, Theorem 5], so we assume that $E$ is non-separable. Suppose that the inclusion $E \subset F$ is not SS and $E \not \supset G$. We have to prove that the inclusion $E \subset F$ is not DSS. Let $Q$ denote the (closed) infinite-dimensional subspace of $E$ on which the norms $\|\cdot\|_{E}$ and $\|\cdot\|_{F}$ are equivalent. Now, if the norms of $E$ and $L_{1}$ were equivalent on $Q$, we would have $E \supset G$, by Theorem 1 of [HNS]. Therefore, we can assume that the norms of $E$ and $L_{1}$ are not equivalent on $Q$.

We first deal with the case of $F$ separable. Consider the real interpolation space $E_{1}:=(E, F)_{\theta, p}$ for some $0<\theta<1$ and $1<p<\infty$. The separability of $F$ implies $\lim _{\text {meas } A \rightarrow 0}\left\|x \chi_{A}\right\|_{F}=0$ for any $x \in F$, so also for $x \in E$. Hence $K(t, x, E, F)=K\left(t, x, E_{0}, F\right)$ for $x \in F$, which implies that $E_{1}=\left(E_{0}, F\right)_{\theta, p}$ (cf. [BL, Thm. 3.4.2]). Therefore $E_{1}$ is also separable and $E \subset E_{1} \subset F$ with $E_{1} \neq F$.

Now, since the norms of $E, E_{1}$ and $F$ are equivalent on $Q$, the norms of $E_{1}$ and $L_{1}$ are not. Hence, applying the Kadec-Pełczyński method ([LT2], see [HNS, Thm. 5]) we can find a normalized sequence $\left\{x_{n}\right\}$ in $Q$ and a sequence of disjoint measurable sets $A_{n} \subset \operatorname{supp} x_{n}, n \in \mathbb{N}$, such that $y_{n}:=$ $x_{n} \chi_{A_{n}} \in L_{\infty}$ and the sequence $\left\{x_{n}\right\}$ is equivalent to $\left\{y_{n}\right\}$ in $E_{1}$ and in $F$. 
Now, using that the fact $\left|\sum c_{n} y_{n}\right| \leq\left|\sum c_{n} x_{n}\right|$ and the equivalence of the norms of $E$ and $E_{1}$ on $\left[x_{n}\right]$, we have

$$
\begin{aligned}
\left\|\sum_{n} c_{n} y_{n}\right\|_{E} & \leq\left\|\sum_{n} c_{n} x_{n}\right\|_{E} \\
& \leq M_{1}\left\|\sum_{n} c_{n} x_{n}\right\|_{E_{1}} \leq M_{2}\left\|\sum_{n} c_{n} y_{n}\right\|_{E_{1}} \leq M_{3}\left\|\sum_{n} c_{n} y_{n}\right\|_{E}
\end{aligned}
$$

for any scalar sequence $\left\{c_{n}\right\}$ and for some constants $M_{1}, M_{2}, M_{3}>0$. Therefore, the sequences $\left\{x_{n}\right\}$ and $\left\{y_{n}\right\}$ are also equivalent in $E$. Thus the norms of $E$ and $F$ are equivalent on $\left[y_{n}\right]$ and the inclusion $E \subset F$ is not DSS.

Finally, assume that $E$ and $F$ are non-separable. We distinguish two cases: $E \subset F_{0}$ and $E \not \subset F_{0}$. If $E \subset F_{0}$, this inclusion cannot be SS and since $F_{0}$ is separable, we deduce as earlier that the inclusion $E \subset F$ is not DSS. In the case of $E \not \subset F_{0}$, we get the same conclusion by proceding as in the second part of the proof of Proposition 1.

Notice that Theorem 2 may be reformulated as follows: the inclusion $E \subset F$ is strictly singular if and only if it is disjointly strictly singular and the norms of $E$ and $F$ are not equivalent on the Rademacher subspace $\left[r_{n}\right]$.

Corollary 3. Let $E$ and $F$ be r.i. spaces with $E \subset F$ and $E \not \supset G$. If the norms of $E$ and $F$ are equivalent on $\left[r_{n}\right]$ then there exists a disjoint sequence $\left\{x_{n}\right\}$ in $E$ for which the norms of $E$ and $F$ are equivalent on $\left[x_{n}\right]$.

Corollary 4. Let $E$ and $F$ be r.i. spaces with $E \subset F$. The inclusion $E \subset F$ is strictly singular if and only if the inclusion $E_{0} \subset F$ is strictly singular.

Proof. The "only if" part is trivial. Suppose that the inclusion $E \subset F$ is not SS. It follows from Theorem 2 that either $E \supset G$, or the inclusion $E \subset F$ is not DSS. If $E \supset G$ then $E_{0} \supset G$ since $G$ is separable. And if the inclusion $E \subset F$ is not DSS then, by Proposition 1, neither is the inclusion $E_{0} \subset F$; all the more, it is not SS.

4. Strict singularity and Rademacher spaces. In this section we study couples of r.i. spaces $E$ and $F$ "close" to $L_{\infty}$ with equivalence of norms on the Rademacher subspace. For that we will make use of some interpolation results.

Given a r.i. space $E$, consider the sequence space $R(E)$ of Rademacher coefficients $\left\{a_{k}\right\}$ endowed with the norm

$$
\left\|\left\{a_{k}\right\}\right\|_{R(E)}=\left\|\sum_{k=1}^{\infty} a_{k} r_{k}\right\|_{E} .
$$

It is easy to check that $R(E)$ is an interpolation space between $\ell_{1}$ and $\ell_{2}$, 
i.e., $R(E) \in I\left(\ell_{1}, \ell_{2}\right)$. Moreover, it is known that the set $I\left(\ell_{1}, \ell_{2}\right)$ is described by the real interpolation method (cf. [LS]). Therefore there exists a Banach lattice $F$ of measurable functions on $[0, \infty)$ with respect to the measure $d t / t$ such that $\min (1, t) \in F$ and

$$
R(E)=\left(\ell_{1}, \ell_{2}\right)_{F}^{K}=\left\{a \in \ell_{2}: K\left(t, a, \ell_{1}, \ell_{2}\right) \in F\right\}
$$

(cf. [BK, Thms. 4.4.5 and 4.4.38]).

We can consider the r.i. space $\widetilde{E}$ associated to $E$ defined by

$$
\widetilde{E}:=\left(L_{\infty}, G\right)_{F}^{K}
$$

with its canonical norm $\|x\|_{\widetilde{E}}=\left\|K\left(\cdot, x, L_{\infty}, G\right)\right\|_{F}$, where $G$ is the closure of $L_{\infty}$ in $L_{N} \equiv \exp L_{2}$. It is known ([A, Thm. 1.4]) that $R(E)=R(\widetilde{E})$, i.e.,

$$
\left\|\sum_{k=1}^{\infty} a_{k} r_{k}\right\|_{\widetilde{E}} \asymp\left\|\sum_{k=1}^{\infty} a_{k} r_{k}\right\|_{E} .
$$

Moreover, $E=\widetilde{E}$ if and only if $E \in I\left(L_{\infty}, G\right)$ ([A, Thm. 1.5]).

Given $x \in L_{N}$, denote by $S x$ the function

$$
S x(t):=\log ^{1 / 2} \frac{e}{t} \sup _{0<u \leq t} x^{*}(u) \log ^{-1 / 2} \frac{e}{u}
$$

for $0<t \leq 1$. The following statement gives a simple description of the r.i. space $\widetilde{E}$.

Proposition 5. If $E$ is an r.i. space then $\|x\|_{\widetilde{E}} \asymp\|S x\|_{E}$.

Proof. It follows from [M, Cor. 2.2] that there exist absolute constants $C_{1}, C_{2}, \beta>0$ such that for all $a \in \ell_{2}$,

$$
\left(\sum_{k=1}^{\infty} a_{k} r_{k}\right)^{*}(t) \leq C_{1} K\left(\log ^{1 / 2}(e / t), a, \ell_{1}, \ell_{2}\right)
$$

and

$$
\left(\sum_{k=1}^{\infty} a_{k} r_{k}\right)^{*}(\beta t) \geq C_{2} K\left(\log ^{1 / 2}(e / t), a, \ell_{1}, \ell_{2}\right)
$$

for every $0<t \leq 1$. Hence

$$
\|a\|_{R(E)} \asymp\left\|K\left(\log ^{1 / 2}(e / t), a, \ell_{1}, \ell_{2}\right)\right\|_{E} .
$$

The sets of $K$-functionals corresponding to the Banach pairs $\left(L_{\infty}, G\right)$ and $\left(\ell_{1}, \ell_{2}\right)$ coincide up to equivalence ([A]). If $x \in \widetilde{E}$, then $K\left(t, x, L_{\infty}, G\right) \in F$ and there exists $a \in l_{2}$ such that

$$
K\left(t, x, L_{\infty}, G\right) \asymp K\left(t, a, \ell_{1}, \ell_{2}\right)
$$

for $t>0$. Hence $a \in R(E)$ and, by (4), we have $K\left(\log ^{1 / 2}(e / t), a, \ell_{1}, \ell_{2}\right) \in E$. Using (5) we get $K\left(\log ^{1 / 2}(e / t), x, L_{\infty}, G\right) \in E$. 
Similar arguments show that the converse holds: if $K\left(\log ^{1 / 2}(e / t), x\right.$, $\left.L_{\infty}, G\right) \in E$, then $x \in \widetilde{E}$. Thus the space $\widetilde{E}$ and the (Banach) space endowed with the norm $\left\|K\left(\log ^{1 / 2}(e / t), \cdot, L_{\infty}, G\right)\right\|_{E}$ coincide as sets, so, by the closed graph theorem, both norms are equivalent, i.e.,

$$
\|x\|_{\widetilde{E}} \asymp\left\|K\left(\log ^{1 / 2}(e / t), x, L_{\infty}, G\right)\right\|_{E} .
$$

Finally, it is easy to see that

$$
K\left(t, x, L_{\infty}, G\right)=K\left(t, x, L_{\infty}, L_{N}\right)
$$

for any $x \in G$ and $t>0$. And it is well known ([Lo]) that

$$
\|x\|_{L_{N}} \asymp\|x\|_{M\left(\varphi_{0}\right)}
$$

for $x \in L_{N}$ where $\varphi_{0}(t)=\log ^{-1 / 2}(e / t)$, and clearly, $L_{\infty}=M\left(\varphi_{1}\right)$ for the function $\varphi_{1}(t)=1$. Therefore we can consider the Banach pair $\left(L_{\infty}, L_{N}\right)$ as a pair of Marcinkiewicz spaces and apply a formula for the $K$-functional from $[\mathrm{CN}]$. Thus we have

$$
K\left(\log ^{1 / 2}(e / t), x, L_{\infty}, L_{N}\right) \asymp \log ^{1 / 2}(e / t) \sup _{0<u \leq t} x^{*}(u) \log ^{-1 / 2}(e / u),
$$

and the needed equivalence follows now from (6)-(9).

Note that $S x(t) \geq x^{*}(t)$. Hence the above proposition implies that $\widetilde{E} \subset E$ and $\|x\|_{E} \leq C\|x\|_{\widetilde{E}}$ for every $x \in \widetilde{E}$ and some constant $C>0$. In particular, $\varphi_{E}(t) \leq C \varphi_{\widetilde{E}}(t)$ for every $t \in[0,1]$.

We can now give a characterization of the Lorentz and Marcinkiewicz spaces which are interpolation spaces between $L_{\infty}$ and $G$.

Proposition 6. Let $\psi \in \Omega$.

(i) A Lorentz space $\Lambda(\psi)$ belongs to the set $I\left(L_{\infty}, G\right)$ if and only if $\varphi \widetilde{\Lambda(\psi)}(t) \leq C \psi(t)$ for some $C>0$ and $0 \leq t \leq 1$.

(ii) A Marcinkiewicz space $M(\psi)$ belongs to the set $I\left(L_{\infty}, L_{N}\right)$ if and only if $\varphi \widetilde{M(\psi)}(t) \leq C \psi(t)$ for some $C>0$ and $0 \leq t \leq 1$.

Proof. (i) If $\Lambda(\psi) \in I\left(L_{\infty}, G\right)$ then $\widetilde{\Lambda(\psi)}=\Lambda(\psi)$ ([A]) and hence the functions $\varphi_{\overparen{\Lambda(\psi)}}$ and $\psi$ are equivalent.

Conversely, if $\varphi \widetilde{\Lambda(\psi)}(t) \leq C \psi(t)$, then Proposition 5 implies that the quasi-linear operator $S$ on $\Lambda(\psi)$ defined in (3) is uniformly bounded on the set of characteristic functions. Hence [KPS, Lemma 2.5.2] shows that $S$ is bounded in $\Lambda(\psi)$. Therefore $\|S x\|_{\Lambda(\psi)} \leq C\|x\|_{\Lambda(\psi)}$ for some $C>0$, and Proposition 5 yields $\widetilde{\Lambda(\psi)}=\Lambda(\psi)$, and hence $\Lambda(\psi) \in I\left(L_{\infty}, G\right)$.

(ii) If $M(\psi) \in I\left(L_{\infty}, L_{N}\right)$ then $M(\psi) \cap G \in I\left(L_{\infty}, G\right)$. Indeed, since the set $I\left(L_{\infty}, L_{N}\right)$ can be described by the real interpolation method ([A]) we have $M(\psi)=\left(L_{\infty}, L_{N}\right)_{F}^{K}$ for some Banach lattice $F$ on $[0, \infty)$. And, by $(7)$, 
we have $M(\psi) \cap G=\left(L_{\infty}, G\right)_{F}^{K}$. This means that $M(\psi) \cap G \in I\left(L_{\infty}, G\right)$ and $M(\psi) \cap G=\widetilde{M(\psi) \cap} G$. Moreover, since $M(\psi) \subset L_{N}$ the fundamental functions of the spaces $M(\psi)$ and $M(\psi) \cap G$ are equivalent, and

$$
\left\|\sum a_{k} r_{k}\right\|_{M(\psi)} \asymp\left\|\sum a_{k} r_{k}\right\|_{M(\psi) \cap G},
$$

therefore $\widetilde{M(\psi)}=\widetilde{M(\psi) \cap}$. Hence,

$$
\varphi_{\widetilde{M(\psi)}}(t) \asymp \varphi_{M(\psi) \cap G}(t) \asymp \varphi_{M(\psi) \cap G}(t) \asymp \varphi_{M(\psi)}(t)=\psi(t) .
$$

Let us now prove the converse. Assume $\varphi_{\widetilde{M(\psi)}}(t) \leq C \psi(t)$. Then, by Proposition 5, there is $C>0$ such that

$$
\left\|S \chi_{(0, \tau)}\right\|_{M(\psi)} \leq C \psi(\tau)
$$

for $\tau \in[0,1]$. Now, since

$$
S \chi_{(0, \tau)}(t)= \begin{cases}1, & 0<t \leq \tau \\ \left(\frac{\log (e / t)}{\log (e / \tau)}\right)^{1 / 2}, & \tau \leq t \leq 1,\end{cases}
$$

and $\int_{0}^{t} \log ^{1 / 2}(e / s) d s \asymp t \log ^{1 / 2}(e / t)$, for $0<t \leq 1$, we get

$$
\begin{aligned}
\frac{1}{t} \int_{0}^{t} S \chi_{(0, \tau)}(s) d s & \asymp \chi_{(0, \tau)}(t)+\left(\frac{\tau}{t}+\left(\frac{\log (e / t)}{\log (e / \tau)}\right)^{1 / 2}\right) \chi_{(\tau, 1)}(t) \\
& \asymp S \chi_{(0, \tau)}(t) .
\end{aligned}
$$

Hence

$$
\left\|S \chi_{(0, \tau)}\right\|_{M(\psi)} \asymp\left(\log \frac{e}{\tau}\right)^{-1 / 2} \sup _{\tau \leq t \leq 1} \psi(t) \log ^{1 / 2} \frac{e}{t} .
$$

Therefore (10) can be rewritten as

$$
\frac{\psi(t)}{\psi(\tau)} \leq C_{1}\left(\frac{\log (e / \tau)}{\log (e / t)}\right)^{1 / 2}, \quad 0<\tau \leq t \leq 1 .
$$

Since $\varphi_{L_{N}}(t)=\log ^{-1 / 2}(e / t)$ the above inequality proves, by [S], that $M(\psi) \in$ $I\left(L_{\infty}, L_{N}\right)$.

Corollary 7. Let $E$ be an r.i. space with $E \in I\left(L_{\infty}, G\right)$. Then the Marcinkiewicz space $M\left(\varphi_{E}\right)$ belongs to $I\left(L_{\infty}, L_{N}\right)$.

Proof. We have $E \subset M\left(\varphi_{E}\right)$ (cf. [KPS, Thm. 2.5.7]) and, using [A] once more, we get $E=\widetilde{E} \subset \widetilde{M\left(\varphi_{E}\right)} \subset M\left(\varphi_{E}\right)$. Now, since $E$ and $M\left(\varphi_{E}\right)$ have the same fundamental functions, the spaces $\widetilde{M\left(\varphi_{E}\right)}$ and $M\left(\varphi_{E}\right)$ have equivalent fundamental functions. This implies, by Proposition 6 above, that $M\left(\varphi_{E}\right) \in I\left(L_{\infty}, L_{N}\right)$. 
Corollary 8. Given a Marcinkiewicz space $M(\varphi)$, there exists a Marcinkiewicz space $M(\psi)$ with $M(\psi) \in I\left(L_{\infty}, L_{N}\right)$ such that $\widetilde{M(\varphi)}=M(\psi) \cap G$.

Proof. Let $\psi$ denote the fundamental function of $\widetilde{M(\varphi)}$. Since $\widetilde{M(\varphi)} \in$ $I\left(L_{\infty}, G\right)$, Corollary 7 yields $M(\psi) \in I\left(L_{\infty}, L_{N}\right)$. Now, from the inclusions $M(\varphi) \subset M(\psi) \cap G \subset M(\varphi)$ and (2), we deduce that

$$
\left\|\sum_{k=1}^{\infty} a_{k} r_{k}\right\|_{\widetilde{M(\varphi)}} \asymp\left\|\sum_{k=1}^{\infty} a_{k} r_{k}\right\|_{M(\psi) \cap G} \asymp\left\|\sum_{k=1}^{\infty} a_{k} r_{k}\right\|_{M(\varphi)}
$$

for all sequences $a \in \ell_{2}$. Since it was proved in Proposition 6 that $M(\psi) \cap G \in$ $I\left(L_{\infty}, G\right)$, an application of [A, Thm. 1.5] shows that $\widetilde{M(\varphi)}=M(\psi) \cap G$.

We are now in a position to present one of the main results of this section.

Theorem 9. If a Marcinkiewicz space $M(\varphi) \subset G$ does not belong to $I\left(L_{\infty}, G\right)$, then there exists another Marcinkiewicz space $M(\psi)$ such that $M(\psi) \subsetneq M(\varphi)$ and $R(M(\varphi))=R(M(\psi))$.

Proof. We have $\widetilde{M(\varphi)} \subset M(\varphi) \subset G$. Hence, by Corollary 8 , if $\psi$ is the fundamental function of $\widetilde{M(\varphi)}$ then $\widetilde{M(\varphi)}=M(\psi) \cap G=M(\psi)$ and $M(\psi) \in I\left(L_{\infty}, G\right)$. Moreover, the Marcinkiewicz spaces $M(\varphi)$ and $M(\psi)$ do not coincide because $M(\varphi) \notin I\left(L_{\infty}, G\right)$. Finally, by (2), we have $R(M(\varphi))=$ $R(M(\psi))$.

An analogous result is also valid for Lorentz spaces.

Theorem 10. If a Lorentz space $\Lambda(\varphi) \subset G$ does not belong to $I\left(L_{\infty}, G\right)$, then there exists another Lorentz space $\Lambda(\psi)$ such that $\Lambda(\psi) \subsetneq \Lambda(\varphi)$ and $R(\Lambda(\varphi))=R(\Lambda(\psi))$.

Proof. Let $X:=\widetilde{\Lambda(\varphi)}$. Since $X \in I\left(L_{\infty}, G\right)$ and $\Lambda(\varphi) \notin I\left(L_{\infty}, G\right)$, we have $X \subsetneq \Lambda(\varphi)$. It is easily checked that the Köthe dual $X^{\prime}$ satisfies $X^{\prime} \supsetneq$ $M(t / \varphi)$. Therefore, there exists a positive decreasing function $a(\cdot) \in X^{\prime}$ such that

$$
a(t) \geq \varphi^{\prime}(t) \quad(0<t \leq 1) \quad \text { and } \quad \limsup _{t \rightarrow+0} \frac{1}{\varphi(t)} \int_{0}^{t} a(s) d s=\infty .
$$

Define $\psi(t):=\int_{0}^{t} a(s) d s(0 \leq t \leq 1)$. Since for every $x \in X$ we have

$$
\int_{0}^{1} x^{*}(t) d \psi(t)=\int_{0}^{1} x^{*}(t) a(t) d t<\infty
$$

it follows that $X \subset \Lambda(\psi)$. Moreover, $\Lambda(\psi) \subsetneq \Lambda(\varphi)$, so the equality $R(\Lambda(\varphi))=$ $R(\Lambda(\psi))$ follows from $(2)$. 
In particular, the above inclusions $M(\psi) \subset M(\varphi)$ and $\Lambda(\psi) \subset \Lambda(\varphi)$ are not strictly singular and, by Corollary 3 , not disjointly strictly singular either. By contrast, we have the following:

TheOREM 11. Let $\varphi, \psi \in \Omega$ be such that $\Lambda(\varphi) \subset M(\psi)$. The inclusion $\Lambda(\varphi) \subset M(\psi)$ is strictly singular if and only if $\Lambda(\varphi) \not \supset G$ and $\psi(+0)=0$.

Proof. The necessity part is well known. If $\Lambda(\varphi) \supset G$ then $R(\Lambda(\varphi))=$ $R(M(\psi))=\ell_{2}([\mathrm{RS}])$. In the case when $\psi(+0)>0$ we have $M(\psi)=L_{\infty}=$ $\Lambda(\varphi)$.

Conversely, since $\psi(+0)=0$ we have $M(\psi) \neq L_{\infty}$, and clearly $\Lambda(\varphi) \neq L_{1}$. Hence the spaces $\Lambda(\varphi)$ and $M(\psi)$ do not coincide. Thus the statement is known for the left extreme case of $\Lambda(\varphi)=L^{\infty}([\mathrm{N}])$ and also for the right extreme case of $M(\psi)=L^{1}$ since $\Lambda(\varphi) \not \supset G$ ([HNS, Thm. 1]). Now, using the fact that any normalized disjoint sequence in $\Lambda(\varphi)(\operatorname{resp} . M(\psi))$ contains a subsequence equivalent to the canonical basis of $\ell_{1}$ [FJT] (resp. of $c_{0}$, cf. [Se]) we deduce that the inclusion $\Lambda(\varphi) \subset M(\psi)$ is DSS. Hence, by Theorem 2, it is also SS.

In particular: the canonical inclusion $\Lambda(\varphi) \subset M(\varphi)$ is strictly singular if and only if $\Lambda(\varphi) \not \supset G$ and $\varphi(+0)=0$.

A direct consequence is

Corollary 12. Let $\varphi \in \Omega$. Then $R(\Lambda(\varphi))=R(M(\varphi))$ if and only if $\Lambda(\varphi) \supset G$ or $\varphi(+0)>0$.

5. Applications. In this section we give some applications of the main results.

Proposition 13. Let $E$ and $F$ be r.i. spaces with $E \not \supset G$. If

$$
\int_{0}^{1}\left(\frac{t}{\varphi_{E}(t)}\right)^{\prime} \varphi_{F}^{\prime}(t) d t<\infty,
$$

then $E \subset F$ and this inclusion is strictly singular.

Proof. It was proved in Theorem 3.1 of [GHSS] that condition (14) implies the inclusion $E \subset F$ and that this inclusion is DSS. Hence, using Theorem 2, we get the statement.

Corollary 14. Let $E$ and $F$ be r.i. spaces such that $\varphi_{E}(t) \geq a \log ^{-\alpha}(e / t)$ and $\varphi_{F}(t) \leq b \log ^{-\beta}(e / t)$ for some $0<\alpha<\min (\beta, 1 / 2)$ and constants $a, b>0$. Then the inclusion $E \subset F$ is strictly singular.

Proof. We may assume that the functions $t / \varphi_{E}(t)$ and $\varphi_{F}$ are concave on $(0,1]$. Then $\left(t / \varphi_{E}(t)\right)^{\prime}$ and $\varphi_{F}^{\prime}$ decrease on $(0,1]$. Now, applying twice the 
property 2.2 .19 in [KPS], we get

$$
\begin{aligned}
\int_{0}^{1}\left(\frac{t}{\varphi_{E}(t)}\right)^{\prime} \varphi_{F}^{\prime}(t) d t & \leq \int_{0}^{1}\left(\frac{t}{a \log ^{-\alpha}(e / t)}\right)^{\prime}\left(b \log ^{-\beta} \frac{e}{t}\right)^{\prime} d t \\
& \leq \frac{b \beta}{a} \int_{0}^{1} \log ^{\alpha-\beta-1} \frac{e}{t} \frac{d t}{t}=\frac{b \beta}{a(\beta-\alpha)}<\infty .
\end{aligned}
$$

By [KPS, Thm. 2.5.7]), the assumption $\alpha<1 / 2$ implies $E \subsetneq G$. Hence the statement follows from the above proposition.

Proposition 15. Let $E$ and $F$ be r.i. spaces with $E \subset F$. If the inclusion $E \subset F$ is disjointly strictly singular then the inclusion operator is weakly compact.

Proof. We can assume that $E \subset F_{0}$. Indeed, otherwise, reasoning as in the proof of Proposition 1 we construct a disjoint sequence $\left\{z_{k}\right\}$ in $E_{0}$ which is equivalent in $E_{0}$ and in $F_{0}$ to the canonical basis of $c_{0}$. So the inclusion $E \subset F$ is not DSS.

Now, let $E \subset F$ with $F$ separable, hence order continuous. Assume that $E \subset F$ is not weakly compact. Consider the real interpolation space $(E, F)_{\theta, p}$ for $0<\theta<1,1<p<\infty$, which is not reflexive by [B, Thm. 3.1]. Hence the lattice $(E, F)_{\theta, p}$ contains a subspace $Q$ isomorphic to $\ell_{1}$ or to $c_{0}$ (cf. [LT2]). Now if $Q$ is isomorphic to $\ell_{1}$, we find, by [B, Prop. 2.3.3], that the inclusion $E \hookrightarrow F$ preserves an $\ell_{1}$-isomorphic copy. In the case of $Q$ isomorphic to $c_{0}$, an analogous statement is also true [Ma, Cor. 4.1]. Now, using ([Me, Thms. 3.4.11-3.4.17]), we deduce that $E \subset F$ also preserves a disjoint $\ell_{1}$-sequence or a disjoint $c_{0}$-sequence.

V. Milman [Mi] posed the following question: Given two Banach spaces $E$ and $F$ and a non-strictly singular operator $A$ from $E$ into $F$, does there exist a complemented subspace $Q$ in $E$ such that the restriction of the operator $A$ to $Q$ is an isomorphism?

We give a negative answer to this question using the above results. First note that the inclusions $L \log ^{\lambda} L \subset L_{1}$ are not strictly singular for $\lambda>0$ because the Rademacher spaces satisfy $R\left(L \log ^{\lambda} L\right)=R\left(L_{1}\right)=\ell_{2}$.

Recall that an operator $A: E \rightarrow F$ between two Banach spaces $E$ and $F$ is said to be strictly cosingular (or Petczyński) if there is no infinitedimensional space $H$ and onto operators $h: E \rightarrow H$ and $g: F \rightarrow H$ such that $h=g A$. Note that this class of operators is somewhat related by duality to strictly singular operators $([\mathrm{P}])$.

THEOREM 16. Let $0<\lambda<1 / 2$. If $Q$ is a subspace of $L \log ^{\lambda} L$ on which the $L \log ^{\lambda} L$-norm and the $L_{1}$-norm are equivalent, then $Q$ is not complemented in $L \log ^{\lambda} L$. 
Proof. Suppose the contrary and denote by $P$ a projection from $L \log ^{\lambda} L$ onto $Q$. There exists a reflexive r.i. space $E$ with $L \log ^{\lambda} L \subset E \subset L_{1}$ ([FS]). Therefore $Q$ is a reflexive subspace of $L_{1}$. It follows from Rosenthal's theorem [R, Thm. 8] that $Q$ embeds isomorphically into $L_{p}$ for some $p>1$, i.e., there exists an operator $T:\left(Q,\|\cdot\|_{L_{1}}\right) \rightarrow L_{p}$ which is an isomorphism onto its image. Set $Z=T(Q)$.

Now, consider the inclusion operator $i: L_{p} \hookrightarrow L \log ^{\lambda} L$ which is not strictly cosingular since there exist onto operators $R=T P i: L_{p} \rightarrow Z$ and $T P: L \log ^{\lambda} L \rightarrow Z$ with $T P i=R$. On the other hand, by Corollary 14, the adjoint operator $i^{*}: \exp L_{\mu} \hookrightarrow L_{p^{\prime}}$ is SS because $\mu>2$ (here $\mu=$ $1 / \lambda$ and $\left.p^{\prime}=p /(p-1)\right)$. Hence, using [P, Prop. 1] we conclude that the inclusion operator $i$ is strictly cosingular, which gives a contradiction. Thus the subspace $Q$ cannot be complemented in $L \log ^{\lambda} L$ (and hence not in $L_{1}$ either).

Note that the assumption $0<\lambda<1 / 2$ is essential since the Rademacher subspace $\left[r_{n}\right]$ is complemented in $L \log ^{\lambda} L$ for $\lambda \geq 1 / 2$ (cf. [LT2, Prop. 2.b.4]).

Acknowledgements. The first and the third authors were partly supported by the Russian Fund of Basic Research grants 07-01-96603-a and 0801-00226-a, respectively. The second author was partly supported by Spanish grants MEC MTM 2008-02652 and S/C PR 07-15837.

\section{References}

[A] S. V. Astashkin, About interpolation of subspaces of rearrangement invariant spaces generated by Rademacher system, Int. J. Math. Math. Sci. 25 (2001), 451-465.

[B] B. Beauzamy, Espaces d'interpolation réels : topologie et géométrie, Lecture Notes in Math. 666, Springer, Berlin, 1978.

[BL] J. Bergh and J. Löfström, Interpolation Spaces. An Introduction, Springer, Berlin, 1976.

[BK] Yu. A. Brudnyı̆ and N. Ya. Krugljak, Interpolation Functors and Interpolation Spaces, North-Holland, Amsterdam, 1991.

[CN] M. Cwikel and P. Nilsson, Interpolation of Marcinkiewicz spaces, Math. Scand. 56 (1985), 29-42.

[FJT] T. Figiel, W. B. Johnson and L. Tzafriri, On Banach lattices and spaces having local unconditional structure, with applications to Lorentz function spaces, J. Approx. Theory 13 (1975), 395-412.

[FS] C. Franchetti and E. M. Semenov, A Hilbert space characterization among function spaces, Anal. Math. 21 (1995), 85-93.

[GHSS] A. García del Amo, F. L. Hernández, V. M. Sánchez and E. M. Semenov, Disjointly strictly-singular inclusions between rearrangement invariant spaces, J. London Math. Soc. 62 (2000), 239-252.

[HNS] F. L. Hernández, S. Ya. Novikov and E. M. Semenov, Strictly singular embeddings between rearrangement invariant spaces, Positivity 7 (2003), 119-124. 
[HR] F. L. Hernández and B. Rodríguez-Salinas, On $l^{p}$-complemented copies in Orlicz spaces II, Israel J. Math. 68 (1989), 27-55.

[KPS] S. G. Krein, Ju. I. Petunin and E. M. Semenov, Interpolation of Linear Operators, Amer. Math. Soc., Providence, RI, 1982.

[LT1] J. Lindenstrauss and L. Tzafriri, Classical Banach Spaces. I. Sequence Spaces, Springer, Berlin, 1977.

[LT2] -, -, Classical Banach Spaces. II. Function Spaces, Springer, Berlin, 1979.

[Lo] G. G. Lorentz, Relations between function spaces, Proc. Amer. Math. Soc. 12 (1961), 127-132.

[LS] G. G. Lorentz and T. Shimogaki, Interpolation theorems for the pair of spaces $\left(L^{1}, L^{p}\right)$ and $\left(L^{q}, L^{\infty}\right)$, Trans. Amer. Math. Soc. 159 (1973), 207-221.

[Ma] M. Mastyło, Interpolation Banach lattices containing no isomorphic copies of $c_{0}$, J. Math. Anal. Appl. 341 (2008), 676-688.

[Me] P. Meyer-Nieberg, Banach Lattices, Springer, 1991.

[Mi] V. D. Milman, Operators of class $C_{0}$ and $C_{0}^{*}$, in: Theory of Functions, Functional Analysis and Applications 10, Kharkov, 1970, 15-26 (in Russian).

[M] S. J. Montgomery-Smith, The distribution of Rademacher sums, Proc. Amer. Math. Soc. 109 (1990), 517-522.

[N] S. Ya. Novikov, Boundary spaces for inclusion map between rearrangement invariant spaces, Collect. Math. 44 (1997), 211-215.

[P] A. Pełczyński, On strictly singular and strictly cosingular operators. I. Bull. Acad. Polon. Sci. Sér. Sci. Math. Astronom. Phys. 13 (1965), 31-36.

[RS] V. A. Rodin and E. M. Semenov, Rademacher series in symmetric spaces, Anal. Math. 1 (1975), 207-222.

[R] H. P. Rosenthal, On subspaces of $L^{p}$, Ann. of Math. 97 (1973), 344-373.

[Se] E. M. Semenov, On the stability of the real interpolation method in the class of rearrangement invariant spaces, in: Function Spaces, Interpolation Spaces, and Related Topics (Haifa, 1995), Israel Math. Conf. Proc. 13, Bar-Ilan Univ., Ramat Gan, 1999, 172-182.

[SH] E. M. Semenov and F. L. Hernández, Strictly singular and disjointly strictly singular inclusions, Dokl. Ross. Akad. Nauk 413 (2007), 461-462 (in Russian).

[S] R. Sharpley, Characterization of intermediate spaces of $M_{\varphi}$, in: Linear Operators and Approximation II (Oberwolfach, 1974), P. L. Butzer and B. Sz.-Nagy (eds.), Birkhäuser, 1976, 205-214.

Department of Mathematics

Samara State University

Samara 443029, Russia

E-mail: astashkn@ssu.samara.ru

Department of Mathematics

Voronezh State University

Voronezh 394006, Russia

E-mail: semenov@func.vsu.ru
Department of Mathematical Analysis Madrid Complutense University 28040 Madrid, Spain E-mail: pacoh@mat.ucm.es

Received October 6, 2008

Revised version December 11, 2008 